\title{
CONTROLE DE QUALIDADE DE DROGAS VEGETAIS DE FARMÁCIAS DE MANIPULAÇÃO DE MARINGÁ (PARANÁ - BRASIL)
}

\section{Quality control of vegetable drugs of the manipulation drugstores of Maringa (Parana, Brazil)}

\author{
Mariana L. Tobias ${ }^{1,2}$; Francine Oliveira ${ }^{1,2}$; Késia Pires de Oliveira ${ }^{1,2}$; Luis Carlos \\ Marques $^{1,3_{*}}$ \\ ${ }^{1}$ Laboratório de Farmacognosia, Departamento de Farmácia e Farmacologia, Universidade Estadual de Maringá - \\ Av. Colombo 5790, bloco T22, 87020-900, Maringá - PR - Brasil \\ ${ }^{2}$ Acadêmicas do curso de graduação de Farmácia da UEM \\ ${ }^{3}$ Professor da disciplina de Farmacognosia da UEM
}

*Autor a quem dirigir a correspondência. Email: luis.marques@apsen.com.br

Recebido em 09/04/2007-Aceito em 27/06/2007

RESUMO: Desenvolveu-se um trabalho de controle de qualidade de drogas vegetais comercializadas em farmácias de manipulação de Maringá de acordo com os parâmetros oficiais, visando atender a legislação vigente. Foram analisadas 92 amostras envolvendo drogas vegetais e extratos secos. Obteve-se, no geral, aprovação em 66 amostras (71.7\%) e reprovação em 26 amostras (28.3\%). As reprovações ocorreram em função de problemas relacionados à identidade (38.5\%), pureza (15.4\%) e qualidade química (46.1\%). Esses resultados apontam à existência de problemas de qualidade específicos em algumas matérias primas vegetais fornecidas às farmácias de manipulação, bem como valorizam as determinações legais para a área.

PALAVRAS-CHAVE: Controle de qualidade, drogas vegetais, farmácias de manipulação.

ABSTRACT: "Quality control of vegetable drugs of the manipulation drugstores of Maringa (Parana, Brazil)". Was made a work of quality control of vegetable drugs marketed at drugstores of manipulation in Maringa in agreement with the official parameters, seeking to assist the effective legislation. Were analyzed 92 samples, being drugs vegetables and dry extracts. It was obtained $71.7 \%$ of approval and $28.3 \%$ of disapproval in function of the aspects identity (38.5\%), purity (15.4\%) and chemical quality (46.1\%). Those results appear to the maintenance of the lack of quality of matters vegetable cousins supplied to the manipulation drugstores, as well as they value the legal determinations for the area.

KEY-WORDS: Quality control, vegetable drugs, manipulation drugstores

\section{INTRODUÇÃO}

A atividade farmacêutica teve origem na preparação artesanal de medicamentos, posteriormente evoluindo para a fase industrial. Por motivos diversos, a atividade da manipulação teve uma retomada de interesse nas últimas décadas e hoje ocupa fatores expressivos de mercado.

Pelo volume de matérias primas associadas a essa atividade e as dificuldades de avaliação da qualidade dos materiais levaram ao surgimento de problemas de diversas ordens, estimulando a regulamentação oficial do segmento para assegurar a qualidade dessa classe de produtos. Para isso, o Ministério da Saúde Brasileiro, através da Agência Nacional de Vigilância Sanitária, editou a Resolução RDC no 33 (BRASIL, 2000) a qual estabeleceu parâmetros das atividades das farmácias de manipulação. No entanto, tal norma foi editada com enfoque predominantemente voltado às boas práticas farmacêuticas e com exigências de controle de qualidade 
específicas para fármacos sintéticos, geralmente inaplicáveis aos produtos da classe de fitoterápicos. Às farmácias, em relação às drogas vegetais, restou a opção da busca dos conceitos clássicos de Farmacognosia como roteiro básico de controle de qualidade.

Com o início das atividades de fiscalização por parte dos serviços municipais de vigilância sanitária, criouse a demanda por tais atividades de avaliação de qualidade. Em Maringá, várias farmácias de manipulação, visando buscar formas de atender à citada legislação, procuraram a Universidade Estadual de Maringá e formalizaram convênio de controle de qualidade de drogas vegetais e produtos fitoterápicos. De um universo de 25 estabelecimentos, 7 deles compuseram o grupo de interessados, formalizaram convênio institucional e iniciaram a remessa de suas amostras.

O presente trabalho relata os primeiros dois primeiros anos dessas atividades de controle de qualidade de drogas vegetais e fitoterápicos.

\section{MATERIAL E MÉTODOS}

Foram analisadas amostras de matéria-prima recebidas das farmácias de manipulação, envolvendo drogas vegetais e extratos secos, de lotes adquiridos de fornecedores diversos. Todos esses lotes foram adquiridos acompanhados de laudo de controle de qualidade, sendo que as farmácias encaminharam os lotes e cópias dos laudos respectivos.

Os recipientes contendo as matérias-primas foram sempre recebidos lacrados e rotulados, não se aceitando outra condição para as embalagens. Avaliava-se inicialmente o rótulo conferindo-se os dados com o laudo do fornecedor; a abertura do recipiente ocorria sempre no momento das análises, procurando-se primeiramente realizar a homogeneização do conteúdo dos frascos.

Procederam-se à avaliação dos aspectos identidade, pureza e qualidade química. Para a avaliação macro e microscópica foi empregado microscópio estereoscópio (lupa) e ótico binocular; foram montadas lâminas semipermanentes com cortes histológicos manuais das respectivas drogas vegetais clarificadas e coradas pelo sistema safranina e azul de Astra (FARMACOPÉIA BRASILEIRA, 1988). As avaliações ocorreram por comparação com as descrições farmacopêicas, com literaturas de macro e microscopia de drogas vegetais (ALQUINI et al 2000; GILG et al, 1942; OLIVEIRA et al, 1991) ou com padrões botanicamente identificados, disponíveis na coleção da disciplina (RABELO et al, 2001) para aquelas drogas sem citação nas farmacopéias ou nas literaturas anteriormente apresentadas.

Os testes de pureza buscaram detectar a presença de matéria orgânica estranha (MOE), bem como de insetos e sujidades diversas, sendo realizados tanto a olho nu quanto com o auxílio de lupa. Para complementação, realizou-se a determinação de cinzas totais e insolúveis em ácido de acordo com técnicas farmacopêicas.

As análises fitoquímicas preliminares, por sua vez, foram realizadas através de testes qualitativos (testes gerais de caracterização de grupos químicos) e testes quantitativos ou semiquantitativos. A realização desses testes seguiu as recomendações descritas em literatura oficial (WORLD HEALTH ORGANIZATION, 1992), em literaturas esparsas (MOREIRA et al, 1979; MATOS et al 1988, DUARTE et al, 1990) ou em comparação com amostras padrão da coleção de drogas da disciplina de Farmacognosia (RABELO et al, 2001).

Os doseamentos realizados nas drogas obedeceram às características químicas de cada uma e envolveram os seguintes testes:

- ácidos cafeoilquínicos = método espectrofotométrico (FARMACOPEA UFFICIALE DELLA REPUBLICA ITALIANA, 1991)

- $\quad$ alcalóides = volumetria de neutralização (FARMACOPÉIA, 1988)

- $\quad$ antraquinonas totais= método espectrofotométrico (FARMACOPÉIA, 1988)

- $\quad$ flavonóides totais= método espectrofotométrico (FARMACOPÉIA, 1988)

- $\quad$ metil-xantinas= método espectrofotométrico (FARMACOPÉIA, 1988)

- $\quad$ óleos essenciais= hidrodestilação em aparelho de Clevenger (FARMACOPÉIA, 1988)

- $\quad$ saponinas totais= método espectrofotométrico (VIGO, et al., 2003)

taninos totais= método espectrofotométrico (GLASL, 1983)

Já as técnicas semiquantitativas envolveram o índice de espuma (DUARTE et al, 1990) e o índice de intumescimento (COSTA, 2000). Realizou-se, também, como fator confirmatório da identidade, cromatografias em camada delgada com placas de sílica gel Merck para os casos das drogas pulverizadas maracujá (FARMACOPÉIA,1988), espinheira santa (FRANCO et al, 1990) e ginseng brasileiro (OLIVEIRA et al, 1980). Por fim, realizou-se também o teor de extrativos aquoso e a perda por dessecação (WORLD HEALTH ORGANIZATION, 1992). 


\section{RESULTADOS E DISCUSSÃO}

\section{Dados das drogas vegetais}

Foram analisadas 92 amostras de fitoterápicos das farmácias de manipulação de Maringá nos anos de 2001-2003, sendo 66 amostras de drogas vegetais rasuradas ou pulverizadas e 26 amostras de extratos secos. Desse total de 92 amostras, 26 apresentaram problemas que levaram à sua reprovação (28.3\%) e 66 amostras foram aprovadas (71.7\%). As amostras de drogas vegetais analisadas e o respectivo número de cada uma constam da Tabela 1.

\begin{tabular}{ll|ll}
\hline \multicolumn{1}{c}{ Espécie } & N & \multicolumn{1}{|c}{ Espécie } & N \\
\hline Abacateiro (Persea gratissima) & 2 & Ginkgo folhas (Ginkgo biloba) & 2 \\
Agar-ágar (Gelidium sp) & 1 & Ginseng brasileiro (Pfaffia paniculata) & 5 \\
Boldo-do-Chile (Peumus boldus) & 2 & Glucomanan (Amorphophallus konjac) & 1 \\
Calumba (Jatrorrhiza palmata) & 1 & Graviola (Anonna muricata) & 1 \\
Carqueja (Baccharis trimera) & 1 & Guaraná (Paullnia cupana) & 4 \\
Carvão vegetal & 1 & Hamamelis tintura (Hamamelis virginiana) & 1 \\
Catuaba (Anemopaegma arvense) & 1 & Levedura (Saccharomyces cerevisiae) & 1 \\
Cavalinha (Equisetum arvense) & 1 & Maracujá (Passiflora alata) & 3 \\
Centella (Centella asiatica) & 3 & Marapuama (Ptychopetalum olacoides) & 2 \\
Chá de bugre (Casearia sylvestris) & 1 & Pata de vaca (Bauhinia forficata) & 1 \\
Chá de Java (Orthosiphon stamineus) & 1 & Porangaba (Cordia ecalyculata) & 7 \\
Chlorella (Chlorella pyrenoidosa) & 1 & Salsaparrilha (Smilax japecanga) & 1 \\
Echinacea (Echinacea purpurea) & 1 & Sene (Cassia senna) & 6 \\
Espinheira-santa (Maytenus ilicifolia) & 4 & Tanaceto (Tanacetum parthenium) & 1 \\
Fucus (Fucus vesiculosus) & 3 & Valeriana (Valeriana officinalis) & 2 \\
Garcínia (Garcinia cambogia) & 1 & Zedoária (Curcuma zedoaria) & TOTAL DE AMOSTRAS \\
\hline Harpagophytum procumbens) & 1 & & 66 \\
\hline
\end{tabular}

Tabela 1. Drogas vegetais analisadas nos dois anos de convênio universidade - farmácias de manipulação.

Desse conjunto destacam-se, pelo volume de amostras enviadas, as drogas porangaba (7 amostras), sene (6 amostras), ginseng brasileiro (5 amostras), espinheira santa e guaraná (4 amostras) e outras em menor número.

Das 7 amostras de porangaba, 5 foram reprovadas por diferenças microscópicas, de perfil cromatográfico e fitoquímicas, comparadas com padrão botanicamente identificado e com características farmacognósticas publicadas (SAITO et al,1986). Esse alto percentual de reprovação deve-se ao fato dessa droga ser a espécie modista em maior destaque nos últimos anos no Brasil como em função de seu pretenso uso para emagrecer; em 
função de tal demanda, o volume consumido deve ter sido maior que o disponível, estimulando o emprego de espécies diferentes como se fossem Cordia ecalyculata, conforme relatos de profissionais da área (MARQUES, 2000).

A Figura 1 mostra o perfil cromatográfico em camada delgada para a porangaba verdadeira (SAITO, et al, 1986) e uma das amostras falsas, onde se verifica a total discrepância entre os perfis das manchas. A espécie 'falsa' deve tratar-se provavelmente de folhas de guaçatonga (Casearia sylvestris), popularmente chamada também de café ou chá de bugre, nomes comuns à espécie porangaba verdadeira.



Figura 1. Cromatografia em camada delgada da planta Porangaba verdadeira Cordia ecalyculata $(P)$ e de amostra comercial (A).

No caso do sene, das 6 amostras enviadas apenas 1 foi reprovada por baixo teor de antracênicos totais e prova A negativa (teste de Bornträeger) (FARMACOPÉIA BRASILEIRA, 1988). Isso evidencia que é uma espécie com baixa ocorrência de problemas de qualidade, confirmando conhecimento farmacognóstico de que os ativos antracênicos são estáveis ao longo do tempo de estocagem e comercialização.

Em relação ao ginseng brasileiro, foram recebidas 5 amostras de raízes pulverizadas, todas identificadas como Pfaffia paniculata. Sabedores de que esse nome geralmente corresponde às raízes de Pfaffia glomerata (Spreng) Pedersen (VIGO et al, 2003), as análises foram realizadas comparando-se as amostras com padrão botanicamente identificado dessa espécie. Em todos os cinco casos avaliados, comprovou-se tal confusão de espécies (Figura 2). De todo modo, visando contribuir ao bom andamento das atividades das farmácias, os laudos foram aprovados como satisfatórios para Pfaffia glomerata, orientando-se as farmácias para que corrigissem seus rótulos para a espécie verdadeiramente presente no produto e para que retornassem tal informação para seus fornecedores visando a alteração para lotes futuros. Ainda em relação a esse produto, todos os laudos que equivocadamente informavam tratar-se de $P$. paniculata, citavam também que as informações 'correspondiam às especificações oficiais da Farmacopéia Brasileira'. Curiosamente, nenhuma das espécies desse gênero consta, até o momento, de qualquer das 4 edições desse compêndio oficial, demonstrando a necessidade de que os laudos evitem expressões padronizadas que nem sempre se aplicam a todas as drogas vegetais. 


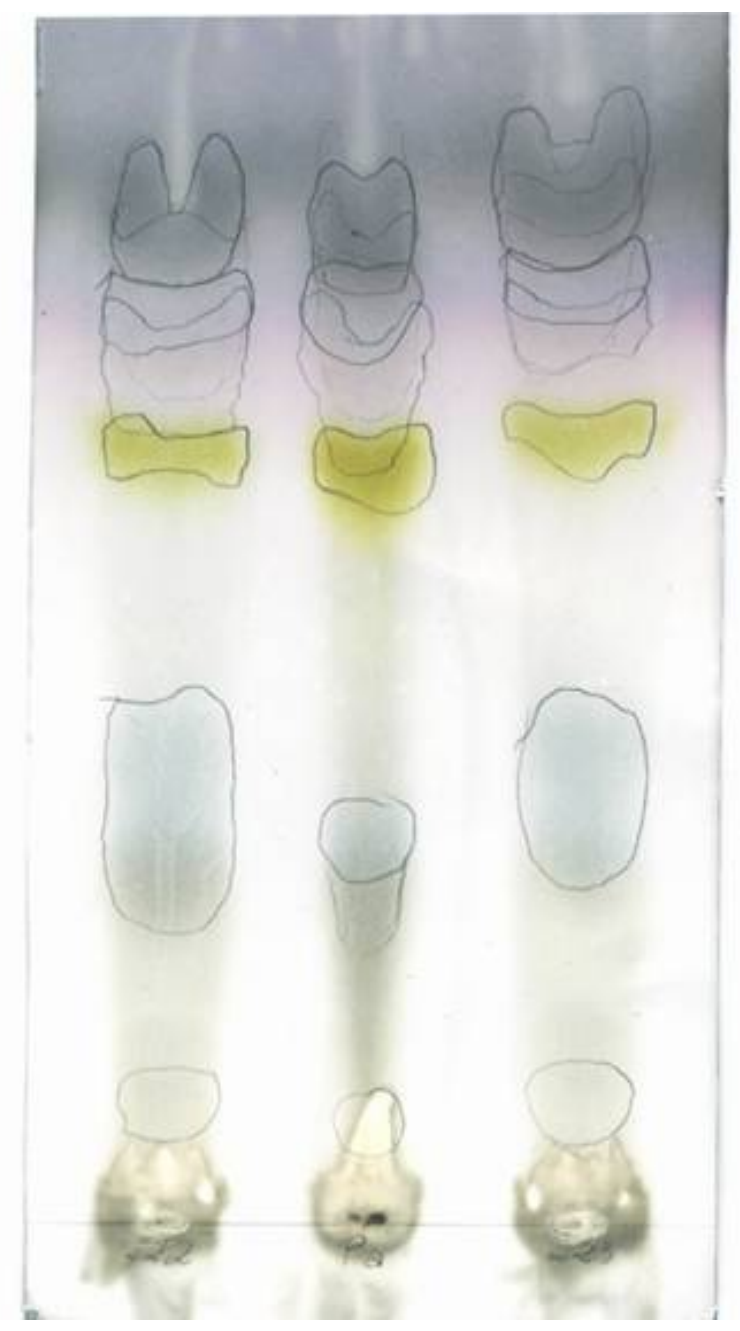

Figura 2. Perfis cromatográficos das amostras 222 e 223 intercaladas pelo padrão botanicamente identificado de Pfaffia glomerata.

Nos casos de espinheira santa, das 4 amostras recebidas 2 foram reprovadas por alto teor de matéria orgânica estranha (lotes com drogas inteiras passíveis de serem avaliadas em relação à presença de caules) e 2 por diferenças microscópicas (lotes rasurados) ou, nos lotes pulverizados, por baixos teores de taninos totais e teor de extrativos aquoso inferior ao estabelecido na literatura (STELFFELD, 1934). Nestes casos, deve estar ocorrendo mistura das folhas com os caules da planta verdadeira, ou ainda a comercialização da espinheira santa falsa (folhas de Sorocea bomplandii - Moraceae) que apresenta folhas espinhosas e é empregada para falsificar a original (ZAUPA et al., 2000).

A droga guaraná teve suas 4 amostras totalmente aprovadas, demonstrando não ter problemas de qualidade no mercado atual, provavelmente por tratar-se de espécie com um bom grau de cultivo na região norte do Brasil e com grupos de ativos quimicamente estáveis.

Outro caso a se destacar é o do maracujá. O mercado fornece lotes rotulados e identificados como Passiflora alata (maracujá doce), espécie inscrita na Farmacopéia brasileira $1^{a}$ edição, mas pouco cultivada no Brasil. Nossas avaliações confirmam que, de fato, os lotes ofertados (3 amostras) correspondem às folhas de Passiflora edulis (maracujá azedo), espécie não farmacopêica mas extensamente cultivada para industrialização de sucos. Trata-se, portanto, de outro caso de adulteração, muito comum e conhecido, mas que vem se mantendo ao longo dos anos. Na verdade, ambas as espécies apresentam estudos farmacológicos que comprovam seus efeitos em casos de agitação nervosa, não havendo a necessidade de empregar-se necessariamente a espécie farmacopêica. Também nesses casos, ofertou-se às farmácias a emissão dos laudos com a correta identificação, orientando-as para que adequassem a rotulagem dos produtos para $P$. edulis e informassem seus fornecedores visando corrigir tais equívocos.

Ainda outros dois casos problemáticos podem ser citados. A droga catuaba atualmente comercializada corresponde majoritariamente às cascas do caule de Trichilia catigua Adr.Juss., cuja identificação e caracterização farmacognóstica foi realizada e publicada na década passada (MARQUES, 1996); no entanto, passados mais de 10 anos desse esclarecimento, continuam sendo fornecidos lotes de catuaba (cascas) com o nome botânico de 
Anemopaegma arvense, espécie inscrita na Farmacopéia e que corresponde a raízes esbranquiçadas e não à cascas, novamente caracterizando situação de fraude. Neste caso, fica clara a influência do mito que representa uma droga constar da farmacopéia, tratando-se de uma distorção que precisa gradativamente ser combatida e modificada. De fato, a correta identificação da espécie e a existência de estudos que comprovem sua eficácia deveriam valer mais do que a simples inclusão no compêndio oficial, ainda mais em se tratando da $1^{\text {a }}$ edição publicada em 1926. Sugere-se, assim, priorizar a comercialização das cascas de Trichilia catigua, atualmente muito mais estudada e com validação farmacológica positiva, se comparada à espécie farmacopêica.

No outro caso, onde deveriam existir raízes da marapuama verdadeira (Ptychopetalum olacoides Benth.), conforme especificações farmacopêicas, encontram-se caules inteiros comercializados como sendo da mesma espécie. Em um dos casos insatisfatórios, a farmácia reclamou ao fornecedor; na resposta (ofício assinado pela farmacêutica responsável) afirma-se a opção pelos caules para não se agredir ecologicamente a espécie com a retirada das raízes. Embora essa informação tenha algum fundamento, caberia aos responsáveis por essa modificação o estudo comparativo entre a parte usada oficial (raízes) e a nova parte proposta para uso (caules inteiros), visando permitir o controle de qualidade bem como confirmar a equivalência química e farmacológica e terapêutica entre essas partes. No entanto, tais dados ainda estão por serem desenvolvidos, representando outro caso problemático em termos de qualidade.

Outro caso interessante é a permanência da comercialização das folhas de Ginkgo biloba (2 amostras), espécie extremamente útil quando empregada na forma de extrato seco padronizado, mas praticamente inútil e desaconselhada na forma de droga vegetal (BLUMENTHAL, 1998). Portanto reforça-se essa orientação de que folhas inteiras ou pulverizadas de Ginkgo biloba não devem ser ofertadas comercialmente por serem inúteis e mesmo prejudiciais aos pacientes.

Em outro aspecto, um dos maiores problemas encontrados ao longo das atividades foi o recebimento de drogas de caráter modista, não-clássicas e para as quais inexistem especificações, oficiais ou não. Estão nessa categoria as drogas: Chlorella, Garcinia e graviola.

Chlorella é um nome genérico de dezenas de espécies de microalgas unicelulares, geralmente recomendadas como imunoestimulantes. Apresenta-se como um pó de cor verde escuro e forte cheiro de 'mar'. Por serem microscópicas, sua diferenciação botânica não é simples e exige especialidade no grupo botânico ou técnicas químicas mais sofisticadas, condições muito difíceis em controle de qualidade nas farmácias de manipulação. Decorre desse contexto a indução de que as matérias primas disponíveis poderiam ser avaliadas apenas ao nível de gênero e sem informações químicas a serem confrontadas, o que evidentemente não é adequado.

Garcinia, por sua vez, corresponde aos frutos de várias espécies desse gênero (Garcinia cola, macrophylla, G. humilis, etc.); como não há especificação farmacognóstica da droga, fica impossível sua verificação nos lotes ofertados comercialmente. Em outro aspecto, além de não se ter ainda confirmação da sua eficácia, os extratos ricos no ativo presente nessa droga já mostram riscos aos pacientes e seu uso deveria ser revisto ou pelo menos minimizado no mercado brasileiro (SAITO et al, 2005; STEVENS et al, 2005).

Graviola é outra droga de uso recente, correspondendo às folhas de Annona muricata (Annonaceae), nas quais se relata a ocorrência de ativos altamente citotóxicos. No entanto, não há estudos com extratos, apenas com ativos isolados, o que impossibilita sua substituição. Em outro aspecto, relata-se a possibilidade do envolvimento dessa espécie em patologias neurodegenerativas (parkinsonismo atípico), (CAPARROS-LEFEBVRE et al, 1999), o que deveria colocá-la como droga experimental mas nunca em amplo processo de comercialização como ocorre no Brasil.

\section{Dados dos extratos secos}

Já em relação aos extratos secos, as análises envolveram 26 amostras de várias drogas vegetais, e sua discriminação é apresentada na Tabela 2.

\begin{tabular}{|c|c|c|c|}
\hline Extrato & $\mathbf{N}$ & Extrato & $\mathbf{N}$ \\
\hline Alcachofra (Cynara scolymus) & 3 & Hypericum (Hypericum perforatum) & 2 \\
\hline Cáscara sagrada (Rhamnus purshiana) & 4 & Kava-kava (Piper methysticum) & 1 \\
\hline $\begin{array}{l}\text { Castanha da Índia } \\
\text { (Aesculus hippocastanum) }\end{array}$ & 4 & Tribulus (Tribulus terrestris) & 1 \\
\hline Garcínia (Garcinia cambogia) & 1 & Isoflavona de soja (Glycine max) & 1 \\
\hline
\end{tabular}


Tabela 2. Extratos secos analisados.

O perfil de amostras de extratos secos é bem diferente do das drogas in natura, pois representam produtos geralmente internacionais, em termos de pesquisa e comercialização. A espécie Ginkgo biloba foi a mais solicitada, pois de fato é o fitoterápico mais utilizado em todo o país e principalmente na forma de extrato seco. A maior dificuldade, no caso dos extratos secos, é a falta de metodologias oficiais de análise e acessíveis a farmácias de manipulação, bem como o alto custo dos padrões necessários à confrontação das amostras.

No caso de matérias primas novas em termos técnicos, como Garcinia ou Tribulus, há quase que absoluta falta de citações em termos de controle de qualidade bem como em relação aos teores mínimos de ativos que seriam esperados na droga, situação que se complica quando se tratam de extratos secos.

\section{Fatores gerais de reprovação}

Em relação aos motivos que levaram à reprovação geral das amostras, tanto drogas como extratos secos, os fatores responsáveis constam da Tabela 3.

\begin{tabular}{lccc}
\hline & Motivos de reprovação & N & $\%$ \\
\hline Identidade & $\begin{array}{c}\text { Diferenças macro, microscópicas ou de perfil } \\
\text { cromatográfico }\end{array}$ & 10 & 38.5 \\
\hline Pureza & Altos teores de MOE ou cinzas & 4 & 15.4 \\
\hline Qualidade química & Baixos teores de ativos & 8 & 30.7 \\
& Diferenças fitoquímicas & 4 & 15.4 \\
\hline
\end{tabular}

Tabela 3. Motivos gerais de reprovação para drogas vegetais e extratos secos (N=26 amostras).

Desses fatores de reprovação, destacam-se os $46.1 \%$ de problemas de qualidade química, principalmente de baixos teores dos grupos de óleos essenciais (especialmente no caso do boldo-do-Chile e zedoária), antracênicos (cáscara sagrada) e mucilagens (ágar e fucus). O caso dos óleos essenciais decorreu do fornecimento da droga pulverizada por parte do atacadista, permanecendo em estoque por alguns meses na farmácia antes da dispensação. Tal procedimento (pulverização) expõe totalmente as essências à temperatura ambiente, levando à sua volatilização e à diminuição brutal e rápida dos teores. Para solucionar esse problema, sugere-se às farmácias que não comprem drogas aromáticas pulverizadas, realizando esse procedimento em sua própria localidade; se isso não for possível, exigir do distribuidor que proceda a pulverização no tempo imediatamente anterior ao envio do lote e colocando-se a menor validade possível nessas matérias primas.

Os problemas de identidade também ocorreram em valores expressivos (38.5\%) e cujos detalhes já foram anteriormente discutidos. No caso de diferenças fitoquímicas, encontram-se evidenciados casos onde os testes fitoquímicos gerais, embora inespecíficos, apontam a grupos cuja presença ou ausência contribui à avaliação final, como ocorreu com uma amostra de extrato seco de unha de gato, com ausência do grupo de ativos principais (alcalóides).

Quanto aos aspectos de pureza, neste caso tiveram baixa expressão (15.4\%). Em estudos anteriores, o maior problema foi altos valores de matéria orgânica estranha (ZAUPA et al, 2000); essa diferença decorre do fato de que o mercado de manipulação trabalha geralmente com drogas vegetais pulverizadas, impossibilitando a verificação da matéria orgânica estranha nos laudos e transferindo ao item "qualidade química" ou "identidade" as diferenças que se manifestam na conclusão final. 


\section{CONCLUSÃO}

Os dados apresentados confirmam que, infelizmente, os problemas de qualidade vêm se mantendo em mercado fitoterápico brasileiro, levando à população prejuízos diversos desde possíveis efeitos colaterais até a ausência dos efeitos benéficos pretendidos com o seu uso. Tais problemas, no entanto, envolvem majoritariamente casos de adulteração/confusão bastante conhecidos e cujo esclarecimento prático é possível com técnicas farmacognósticas básicas perfeitamente exeqüíveis nas farmácias de manipulação. Com base nessa verificação, sugere-se que essas matérias primas sejam priorizadas em termos de currículo farmacêutico de graduação ou em cursos de especialização, visando preparar os farmacêuticos para detectar e eliminar tais problemas.

Em outro aspecto, a simples edição da Resolução 33 (BRASIL, 2000) não se mostrou eficaz na eliminação de tais problemas, talvez decorrente da quase ausência de determinações adequadas à categoria dos fitoterápicos. A edição recente da Resolução 214 (BRASIL, 2006), direcionada à reformulação da norma legal anterior, embora tenha ampliado significativamente seu conteúdo e grau de rigidez, infelizmente pouco acrescentou em termos de aspectos voltados às drogas vegetais e produtos fitoterápicos. Em seu anexo I, item 7.3.13, essa norma exige alguns testes mínimos como caracteres organolépticos, determinação de materiais estranhos, macro e microscopia e ainda densidade para matérias primas líquidas. Como foi mostrado, tais testes feitos isoladamente fornecem dados inconclusivos, ainda mais para espécies de mesmo gênero, lamentando-se a falta da recomendação de cromatografia em camada delgada e dos testes semiquantitativos, atualmente quase desconhecidos e utilizados.

Sugere-se, portanto, alguma mobilização visando sensibilizar os órgãos sanitários a corrigir esses problemas ou mesmo buscar normas específicas à área. Pelos dados anteriormente expostos, só podemos concluir que as normas legais atuais não vêm sendo totalmente efetivas em se tratando de drogas vegetais e extratos secos, mantendo a exposição dos consumidores aos mais diversos tipos de problemas de qualidade.

\section{REFERÊNCIAS BIBLIOGRÁFICAS}

ALQUINI, Y.; TAKEMORI, N. K. Organização estrutural de espécies vegetais de interesse farmacológico. Curitiba: Herbarium, 2000.

BLUMENTHAL, M. The Complete German Commission E monographs. Austin: American Botanical Council, 1998.

BRASIL. Agência Nacional de Vigilância Sanitária. Resolução da Diretoria Colegiada $n^{\circ} 33$ de 19.04.2000. Aprova o Regulamento Técnico sobre Boas Práticas de Manipulação de Medicamentos em farmácias e seus Anexos. Diário Oficial da União, 20.04.2000.

BRASIL. Agência Nacional de Vigilância Sanitária. Resolução da Diretoria Colegiada no 214 de 12.12.2006. Dispõe sobre Boas Práticas de Manipulação de Medicamentos para uso humano em Farmácias. Diário Oficial da União, 18.12.2006.

CAPARROS-LEFEBVRE, D. \& ELBAZ, A. Possible relation of atypical parkinsonism in the French West Indies with consumption of tropical plants: a case-control study. Caribbean Parkinsonism Study Group. Lancet, v. 354, p. 2816, 1999.

COSTA, A. F. Farmacognosia. v. 3. Lisboa: Fundação Calouste Gulbenkian, 2000.

DUARTE, M.R. \& DEBUR, M.C. Stem and leaf morphoanatomy of Maytenus ilicifolia. Fitoterapia, v. 76, p. 41-9, 2005.

DUARTE, M. R., YASSUMOTO, Y. \& CECY, C. Guia de Farmacognosia Aplicada. Curitiba: Educa, 1990.

FARMACOPÉIA BRASILEIRA. $4^{\mathrm{a}}$ ed. São Paulo: Atheneu, 1998.

FARMACOPEA UFFICIALE DELLA REPUBBLICA ITALIANA. ga ed. Roma: Istituto Poligrafico Execca Dello Stato, 1991.

FRANCO, S.L. Maytenus ilicifolia Martius ex. Reiss.- Celastraceae: proposta tecnológica de macerados. 1990. 132p. Dissertação de Mestrado. Escola de Farmácia, Universidade Federal do Rio Grande do Sul, Porto Alegre.

GILG, E., BRANDT, W. \& SSHURHOFF, P. N. Farmacognosia: matéria farmacéutica vegetal y animal. Barcelona: Labor, 1942. 
GLASL, H. Zur photometrie in der drogenstandardisierung-3. Gehaltsbestimmung von gerbstoffdorgen. Deutscher Apotheker Zeitung, v. 123, n. 42, p. 1979-1987, 1983.

MARQUES, L.C. Contribuição ao esclarecimento da identidade botânica da droga vegetal catuaba. Revista Racine, v. 8, n. 43, p. 8-11, 1996.

MARQUES, L.C. Informação pessoal: relato de um farmacêutico do ramo atacadista de drogas vegetais. São Paulo, 2000.

MATOS, F.J. A. Introdução à fitoquímica experimental. Fortaleza: Editora da Universidade Federal do Ceará, 1988.

MOREIRA, E. A. Marcha sistemática de análise em fitoquímica. Tribuna Farmacêutica, v. 47: separata, 1979.

OLIVEIRA, F., AKISUE, G. \& AKISUE. M. K. Contribuição para o estudo farmacognóstico do "ginseng brasileiro" Pfaffia paniculata (Martius) Kuntze. Anais de Farmácia e Química de São Paulo, v. 20, n. 1/2, p. 261-77, 1980.

OLIVEIRA, F., AKISUE, G. \& AKISUE, M. K. Farmacognosia. São Paulo: Atheneu, 1991.

RABELO E SILVA, P. E., MUNDO, S.R., ROCHA, J.C.B., DE SOUZA, D.R. \& MARQUES, L. C. A coleção de drogas como instrumento didático à disciplina de Farmacognosia. In: Anais do III Simpósio Brasileiro de Farmacognosia, Curitiba: Universidade Federal do Paraná, 2001. p. FB13-FB13.

SAITO, M.L. \& OLIVEIRA, F. Morfodiagnose e identificação cromatográfica em camada delgada de chá de bugre Cordia ecalyculata Vell. Revista Brasileira de Farmácia, v. 67, p. 1-16, 1986.

SAITO, M.; UENO, M., OGINO, S., KUBO, K., NAGATA, J. \& TAKEUCHI, M. High dose of Garcinia cambogia is effective in suppressing fat accumulation in developing male Zucker obese rats, but highly toxic to the testis. Food Chem Toxicol., v. 43, p. 411-9, 2005.

STELLFELD, C.A. Espinheira santa: contribuição ao estudo farmacognóstico. Bol. Assoc. Bras.Pharm., v. 15, p. 551-571, 1934.

STEVENS, T., QADRI, A. \& ZEIN, N. N. Two patients with acute liver injury associated with use of the herbal weight-loss supplement hydroxycut. Ann Intern Med., v. 142, p. 477-8, 2005.

VIGO, C.L.S., NARITA, E. \& MARQUES, L. C. Validação da metodologia de quantificação espectrofotométrica das saponinas de Pfaffia glomerata (Spreng.) Pedersen - Amaranthaceae. Revista Brasileira de Farmacognosia, v. 13(supl 2), p. 46-49, 2003.

WORLD HEALTH ORGANIZATION. Quality control methods for medicinal plant materials. Geneva: WHO, 1992.

ZAupA, C., CARRASChI, L., SILVA, E. A., ChANKE, A. L. S., USHIROBIRA, T. M. A. \& MARQUeS. L. C. Controle de qualidade farmacobotânico e legal de fitoterápicos comercializados nas farmácias de Maringá (PR). Revista Racine, v. 10, n. 58, p. 32-38, 2000. 